\title{
A REMARK ON $\varphi-\left|\bar{N}, q_{n} ; \delta\right|_{k}$ SUMMABILITY
}

\author{
MEHMET Ali SARIGÖL
}

Abstract. In a quite recent paper Özarslan [2] has tried to get necessary and sufficient conditions in order that every $\varphi-\left|\bar{N}, p_{n} ; \delta\right|_{k}$ summable series should be $\varphi-\left|\bar{N}, q_{n} ; \delta\right|_{k}$ summable, which extends a result of Bor [1], but does not finalize. In this paper we have solved this open problem.

Mathematics subject classification (2010): 26D15, 40F05, 40G05, 46A45. mappings.

Keywords and phrases: Absolute weighted mean, equivalence methods, sequences spaces, matrix

\section{REFERENCES}

[1] H. BoR, Some equivalence theorems on absolute summability methods, Acta Math. Hungar 149, (2016), 208-214.

[2] H. S. ÖZArslan, Some equivalence theorems on absolute summability methods, Inter. J. Anal. Appl. 13, (2017), 93-97.

[3] M. A. SARIGÖL, On the local properties of factored Fourier series, Appl. Math. Comput. 216, (2010), 3386-3390. 